\title{
La mort dans la doctrine de saint Augustin
}

Le biocosmos se caractérise entre autres par cela que la vịe y est indissolublement accouplée à la mort. L'homme, en tant que partie intégrante du monde animé, est soumis à la loi de la destruction et de la mort. II n'est donc pas étonnant que la problématique de la mort soit un thème durable de l'art, de la littérature, de la philosophie, de la religion et de la. science. Nombreux sont les penseurs qui, avec Platon à leur tête, définissaient la philosophie comme meditatio mortis. Le motif de la mort. Le motif de la mort apparraissait par maintes fois dans les écrits du platonicien chrétien, St. Augustin (354-430). Dans la littérature mondiale, il y a peu de descriptons aussi poignantes que ces fragmens des Confessions où l'auteur représente sa douleur après la perte de son ami de jeunesse. Sa mort a été un choc psychique pour le jeune Augustin qui existentialement avait ressenti l'inévitabilité du départ de ce monde. Dans les Confessions, nous lisons ces paroles émouvantes: "Cette douleur enténébra mon coeur, et partout je ne voyais que mort. La patrie m'était un suplice, la maison paternelle un étrange tourment, tout ce que j'avais partagé avec lui s'était tourné sans lui en torture atroce. Mes yeux le réclamaient de tous les côtés, et on me le donnait pas, et je haissais toutes choses, parce qu'elles ne l'avaient pas et ne pouvaient plus me dire: 'Le voici, il va venir', comme quand il vivait et qu'il était absent. J'étais devenu moi-même pour moi une immense question" '. La mort de l'ami a posé devant Augustin, avec toute son acuité, le problème du sens de la vie et du sens de la mort humaine. Plus tard, il avait vécu la mort de sa mère, Monique ${ }^{2}$. Mais alors, il était déjà préparé spirituellement à la réception de ce fait dans la perspective de l'eschatologie chrétienne.

1. Confes. 4,4,9 PL 32,697. Bibliothèque Augustinenne. Oeuvres de St. Augustin, t. XIII. Trad. de E. Tréhorel et G. Bouissou, Paris 1962, Desclée de Brouwer, p. 421,423.

2. Voir: Confes. 9,11,27-28 PL 32, 775-776. 
La thématique de la mort est un motif relativement fréquent des écrits de St. Augustin, aussi peut étonner le fait que la littérature consacrée à ce thème soit si pauvre. J. Choron, l'auteur de la monographie Death and Western Thought, a complètement omis le penseur chrétien antique ${ }^{3}$. Quelques auteurs ont abordé le problème de la mort chez St. Augustin dans le contexte d'autres problèmes: M. N. Beyenka discutait le problème de la consolation", R. Florez écrivait sur la mort et l'immortalité ${ }^{5}$, J. Volta s'intéressait à. la relation entre la mort et la rédemption ${ }^{6}$. Les analyses de ces auteurs, bien que précieuses, - surtout celles de Flolrez, - n'épuisent pas tous les moments essentiels de la pensée philosophique $e \hat{i}$ théologique d'Augustin. C'est pourquoi il semble utile de revenir sur ce thème. Dans notre article, nous nous intéresserons à la notion de la mort (I), au problème de sa genèse (II), au sens de la mort (III), et au problème de l'attitude chrétienne envers elle (IV).

\section{La notion de la mort}

Les écrits de l'Evêque d'Hippone contiennent la différenciation de la mort physique et le mort spirituelle. La première signifie la mort du corps, la seconde - la mort de l'âme?. Actuellement; il s'agit de la mort comprise en son sens prop're, c'est - à - dire de la mort physique. Augustin l'envisageait aussi bien sur le plan philosophique que théologique, mais les analyses concernant l'aspect biologico-médical font défaut. Probablement, son savoir du domaine médical était assez pauvre ${ }^{8}$, c'est pourquoi les déterminations concernant le phénomène de la mort humaine sous l'aspect médical manquent. L'asse'rtion que la mort.est la perte du sentiment et de la conscience, ne dépasse pas le cadre de l'expérience courante ${ }^{9}$. Assez sommaire est une autre définition de la mort, comme privatio vitae ${ }^{10}$. Cette caractéristique négative de la

3. New York 1963. L'auteur ne mentionne St. Augustin que deux fois, en indiquant la convergence de son idée avec celles d'Heidegger.

4. Consolation in Saint Augustin, Washington 1950.

5. Muerte e inmortalidad en el pensamiento de San Agustín, "La Ciudad de Dios" 174/1961/, p. 449-482.

6. La redenzione della morte nel christiano secondo S. Agostino, Roma 1956-1957.

7. De civ. Dei 13,2 PL 41, 377.

8. Voir: BA, t. XLVIII, Paris 1972, p. 710-714.

9. Trin. 13,8,11 PL 42, 1022.

10. Hypomnesticon contra Pelag. et Coelest. 1,4,5 PL 45,1616. 
mort est à la limite du savoir pré-scientifique et de la philosophie. La mort est la privation de la vie, la lésion de quelque chose d'essentiel dans la nature humaine. Elle est même un anéantissement relatif de l'homme, et notamment de son élément matériel-biologique ${ }^{11}$.

St. Augustin, en déterminant le phénomème de la mort de l'homme, la définissait le plus souvent en tant que séparation de l'âme et du corps. "Mors nempe... separatio est animae a corpore" 12. Cette définition de la mort est liée, comme il semble, à la théorie d'Aristote du hylémorphisme. La notion de "séparation" est analogue à la notion d' "union" de l'âme au corps dans la totalité de la personne humaine, c'est pourquoi la mort dans les différentes conceptions de l'homme est comprise différemment. Certains énoncés du penseur chrétien antique ont un profil platonicien. Lorsqu'il décrivait la mort de sa mére, il employa la formulation suivante: Cette âme religieuse et pieuse se détacha du corps" ${ }^{13}$. A une autre occasion il se servit d'une expression totalement platonicienne: "Mors... id es ab hoc corpore omnimoda fuga et elapsio" ${ }^{14}$. Une telle expression se lie à l'anthropologie de Platon, dans l'interprétation duquel le corps est "une prison" pour l'âme. Dans la conception augustinienne de l'homme, il existe sans doute des éléments de platonisme, néanmoins l'expression ci-dessus est plutôt à comprend're en catégories éthiques et religieuses qu'en catégories ontologiques. Caractéristique est son commentai,re au Livre de la Genèse, où Augustin délimitait l'était de l'homme avant le péché originel et celui après le péché ${ }^{15}$. A l'époque première, c'est-à-dire avant le péché des premiers parents, l'union du corps et de l'âme était quelque chose de naturel. Sous cet égard, la séparation de l'âme et du corps, aussi bien temporaire que d'autant plus, définitive, n'était point désirable. A cause de cela, également, la vérité révélée de la résurrection des corps fut si volontiers acceptée parr le christianisme. Avant le péché originel, le corps n'était donc pas "une prison" pour l'âme, mais présentait un composant naturel de la pelrsonne humaine. "Servitude" de l'âme n'est pas le corps,

11. Voir: De lib. arb. 3,6,18-19 PL 32, 1281.

12. Enar. in ps. 48, sermo 2,2 PL 36,556. Voir: De civ. Dei 13,6 PL 41, $381 ;$ De consolatione mortuorum, sermo 2,1 PL 40,1163 .

13. Confes. $9,11,28$ PL 32,776 .

14. De quant. an. 33,76 PL 32,1077.

15. De Gen. ad litt. 7,27,38 PL 34,369; 12,35;68 PL 34,483-484. 
mais le péché. Les conséquences négatives du péché concernent aussi bien l'âme que le corps ${ }^{16}$. A la lumière de ces explications, il faut comprendre la définition augustinienne de la mort en tant que "libération" ou "fuite" du corps.

La mort, en tant que séparation de l'âme et du corps, entraîne la lésion des structulres essentielles de la personne humaine, et avant tout, la biologico-physique décomposition du corps. Augustin, en apercevant l'analogie entre la mort physique et la mort spirituelle de l'homme, affirme: "Or la mort de l'âme c'est l'impiété, et la mort du corps c'est la corlruptibilité qui entraîne la séparation de l'âme d'avec le corps. L'ame abandonnée de Dieu meurt; ainsi meurt le corps abandonné par l'âme. L'une perd la sagesse et l'autre perd la vie" ${ }^{17}$. Et donc la mort est pourr St. Augustin sépalration de l'âme d'avec le corps, anéantissement du corps matériel, "libération" de l'âme, perte de la conscience, négation et absence de vie. La majorité de ces définitions conçoivent le phénomène de la mort dans l'aspect philosophico-ontologique, ceriaines dans l'aspect éthique.

St. Augustin caractérisait le phénomène de la mort humaine aussi dans l'aspect psychologique-phénoménologique. Ce profil est spécialement distinct dans le "De civitate Dei", où nous trouvons intéressante analyse des termes: vivant, mourant, mort ${ }^{18}$. Au cour de ses considérations, l'auteur affirme que "Les hommes seront, non avant la moirt ni apirès, mais toujours dans la mort; et dès lors, jamais vivants, jamais morts, mais toujours mourants. Jamais, en effet, i! n'y aura pour l'homme de pire malheur dans la mort, que là où la mort elle-même sera immortelle" ${ }^{19}$. Le dernielr fragment de l'énoncé concerne la mort spirituelle, mais en principe, l'analyse présente une large importance du phénomène de la mort physique de l'homme. L'homme, en tant que possesseur d'un coirps matériel, est toujours dans la perspective de perte de la vie ou devant la mort. "En fait, dès l'instant où l'on commence à vivre en un corps

16. "Potest dici et corpus nostrum carcer, non quia carcer est quod Deus fecit, sed quia paenale et mortale". Enar. in ps. 146, 18 PL 37, 1843. 17. Trin. 4,3,5 PL 42, 890, BA, t. XV. Trad. M. Mellet et Th. Camelot Paris 1955 , p. 349 .

18. De civ. Dei 13,11,1-2 PL 41, 384-385.

19. De civ. Dei $13,11,2$ PL 41,$385 ; \mathrm{BA}$, t. XXXV. Trad. B. Dombart et A. Kalb, Paris 1959 , p. 281. 
destiné à mourir, il n'est aucun acte qui ne soit un acheminement vers la mort. Car l'effet de l'instabilité du corps durant toute cette... est de tendre vers la mort" ${ }^{20}$. En sommant ses réflexions, Augustin affirme: "Nihil sit aliud tempus vitae huius, quam cursus ad mortem" 21. La mort n'est donc pas uniquement le moment de la fin de la vie, mais un processus, la totalité de la vie humaine de son essence se diyigeant vers la fin. Une telle conception de la mort se sépare des explications purement biologiques, en attirant l'attention sur le profil psychique-personnel de ce phénomène. Les énoncés d'Augustin rappellent les écrits de $M$. Heidegger, qui définissait la vie humaine comme "Sein-zum-Tode" ${ }^{22}$. Les analyses de lauteur de "Sein und Zeit", concernant la problématique de la mort, sont en une mesure sérieuse une répétition et une continuation des analyses se trouvant dans De civitate Dei et dans les autres oeuvres de l'Evêque d'Hippone. Les deux penseurs, le chrétien antique et le moderne, concevaient le phénomène de la mort en catégories ontologiques et psychologiques, en voyant en elle la perspective durable de la vie humaine. La mort appartient à la condition humaine, sa perspective iréelle accompagne l'homme depuis le moment de sa naissance.

Dans les écrits du penseur chrétien antique, le phénomène de la mort, à côté de l'aspect philosophique et psychologique, apparaît aussi dans l'aspect théologique. Le dernier aspect concerne le plan surnaturel de la vie de l'homme, lié à la possession de la grâce et détruit pair le péché. C'est la seconde mort (mors secunda) qui est separatio animae a Deo ${ }^{23}$. La notion de la "seconde mort" était employée par Lactance et d'autres auteurs du christianisme précoce. Maintes fois, St. Augustin se servait de cette notion, en lui donnant diverses nuances de signification ${ }^{24}$. Ce terme signifie parfois l'état de péché et la perte de la grâce par un homme vivant ${ }^{25}$. Une autre fois, il s'agil de rupture définitive de l'homme avec Dieu, dans la conséquence de quoi,. "l'âme séparée de

20. Ibidem 13,10 PL 41,383; BA, t. XXXV, p. 271.

21. Ibidem.

22. Sein und Zeit, Halle 1941, p. 234,240,245,247.

23. Enar. in ps. 48, sermo 2,2 PL 36, 556.

24. Voir: J. C. Plumpe, Mors secunda, dans: Mélanges Joseph de Ghellinck, Gembloux 1951, t. I, p. 387-403; BA, t. XXXV, p. 526-529.

25. "Mortui sunt omnes infideles, omnes iniqui; corpore vivunt, sed corde exstincti sunt". Enar. in ps. 70, sermo 2,3 PL 36,893 . 
Dieu, mais unie au corps, subit des peines éternelles" ${ }^{26}$. Le sens théologique de la mort, bien qu'essentiel, pour Augustin, joue un rôle secondaire dans nos analyses.

11. Genèse de la mort

Le phénomène de la mort possède un caractère universel. Augustin affirme, que ces hommes uniquement vont l'éviter, qui viviont au dernier moment de l'existence du monde ${ }^{27}$. Ce penseur, en parlant de la genèse de la mort, a différencié ses doubles causes: naturelles et théologiques. Les causes naturelles sont liées à la nature de la personne humaine comme totalité psychophysique. Le commentaire au Livre de la Genèse comprend une assertion caractéristique et lapidaire: "Moirtalis ergo erat conditione corporis animalis, immortalis autem beneficio Conditoris" ${ }^{28}$. L'homme, en tant qu'être corporel-matériel, est mortel. Ainsi est la loi de la nature, que ce qui est matériel et animé, est soumis au processus de vieillissement et de dépérissement. L'immortalité du corps a été l'un des dons extraordinaires que Dieu avait accordé aux premiers hommes. De la liaison organique de la nature et de l'homme avec la mort parle le traité De Trinitate où nous lisons: "Quant au corps, qui est comme 'l'homme extérieur' plus cette vie présente se prolonge et plus il se corrompt sous l'effet de l'âge, de la maladie, de tant de peines, jusqu'à la dernière enfin, qui pour tout le monde n'a qu'un nom: la mort" ${ }^{29}$. La mort, ainsi que l'époque de la vieillesse, ses maux physiques, sont la conséquence de la matério-biologique dimensión de l'homme. "Au sujet du corps- ce n'est pas tant la disparition de l'âme que la précarité de la chair et du sang qui le destine à la mort" ${ }^{30}$. Les énoncés ci-dessus prouvent, que St. Augustin concevait le problème de la mort —son universalité et sa nécessité- aussi sur le plan naturel-ontologique. L'homme meurt, car son corps matériel et animé est soumis aux processus de développe-

26. De civ. Dei 13,12 PL 41,386 .

27. Ibidem 20,20,2-3 PL 41, 688-689.

28. De Gen. ad litt. 6,25,36 PL 34,354 .

29. Trin. 4,3,5 PL 42,890; BA,t.XV, p. 349.

30. Ibidem; BA,t.XV, p. 351 . 
ment et de décomposition. L'homme, conçu comme partie de la nature, ne peut ne pas mourir. La moirt est liée à la nature humaine.

La notion de la nature chez St. Augustin se présente en plusieurs acceptions. D'abord, il faut distinguer la nature de l'homme d'avant le péché originel, et celle d'après. D'abord existait la nature "première" qui comprenait —en outre des qualités naturellesies dons extraordinaires de Dieu. Le péché a affaibli les possibilités de la nature humaine-c'est la seconde nature ${ }^{31}$. Augustin, en pensant à l'étape de la "première" nature, écrivait: "Il est donc clair pour les chrétiens sincèrement attachés à la foi catholique que la mort même du corps ne nous a pas été infligée par une loi de nature" "22. Ce n'est pas là une contestation de la thèse, _précédemment acceptée- que la mort est une conséquence de l'existence du corps matériel dans le cadre de la personne humaine. L'auteur pense uniquement à la nature humaine, gratifiée par Dieu d'une série de privilèges, et entre autres, aussi de celui de l'immortalité du corps. Or le péché des premiers parents a entraîné l'annulation des dons extraordinaires et c'est ainsi que l'on peut dire que la mort est conséquence du péché ${ }^{33}$. La vie immortelle était un don de Dieu, cependant la mort était la peine pour le péché commis ${ }^{34}$. Et donc, elle n'est pas l'oeuvre de Dieu, mais de l'homme. Augustin affirmait aussi que la mort -en tant que privatio vitaene peut venir de Dieu, qui est cause efficiente de natures positives, et non de privations ${ }^{35}$.

L'Evêque d'Hippone, eñ admettant que la mort du corps était ia conséquence du péché originel, introduisit la différenciation entre la "mortalité" et la "mort". Adam -comme homme et possesseur d'un corps matériel-, était mortel, mais n'était pas obligé de mourir ${ }^{36}$. Dans sa nature contingente; il y avait la possibilité de

31. De dic. quaest. ad Simpl, 1,11 PL 40,107; De lib. arb.3,19,54 PL 32, 1297; voir: Ch. Boyer, La notion de nature chez saint Augustin, dans Essais anciens et nouveaux sur la doctrine de saint Augustin, Milano 1970 , p. 215-228.

32. De civ. Dei 13,15 PL 41,387; BA, 2.XXXV, p. 287.

33. Trin. 3,3 PL 42,872; De civ. Dei 13,2 PL 41, 377-378.

34. Retract. $1,21,2$ PL 32,618 .

35. "Mors itaque privatio vitae est, nomen tantum habens, non essentiam: et ideo Deus eius auctor esse dici non potest. Quidquid enim Deum fecisse dicimus, habet essentiam, id est, species est": Hypomnesticon contra Pelag: et Coelest. 1,4,5 PL 45,1616. 
mourir, mais en état d'une nature privilégiée par Dieu, elle ne se serait jamais réalisée. Si donc if n'avait pas commis le péché, il ne serait pas mort, c'est-à-dire son corps ne serait pas séparé de l'âme et il ne serait pas soumis à la putréfaction.

Conséquence du péché, outre la mort, sont aussi les souffrances physiques et psychiques de l'homme ${ }^{37}$. D'habitude, elles précèdent le moment de la mort, et accompagnent le vie humaine. La souffrance peut s'intensifier, disparaître ou se renouveler, mais son existence n'est pas liée inévitablement au fait de la mort ${ }^{38}$. Augustin accentuait la différence des phénomènes de la souffrance et de la mort. Les deux phénomènes appartiennent à la condition de la nature humaine, affaiblie par le péché originel.

\section{Le sens de la mort}

Si la mort est en fait la destinée de l'homme, la question essentielle se pose: est-elle un non-sens global et manifeste, ou est-elle d'une manière quelconque, sensée? St. Augustin, comme chrétien, reconnaissait le sens de la vie humaine. Si donc elle doit inévitablement finir par la mort du corps, alors indirectement il semblait aussi reconnaitre le sens de la mort. Mais peut-on parleir d'un quelconque sens de la mort, qui est anéantissement de la personnalité humaine et fin de sa durée en structure précédente?

Actuellement célèbre est la théorie de l' "option finale". A ses partisans principaux appartiennent L. Boros, J. Pieper et R. Troisfontaines. Ils soutiennent que l'homme est, au moment de la mort, enfin capable de prendre une pleinement consciente et entièrement libre "dernière décision" envers Dieu. Dans cette interprétation, la mort doit être le moment culminant de l'évolution de "l'homme intérieur", bien qu'elle soit en même temps anéantissement de "l'homme extérieur". Boros, en justifiant son attitude envers la nature et le rôle de la mort, se réfère de St. Augustin ${ }^{2}$. Ses écrits

\footnotetext{
36. "Sed ipsum mortale non est factum mortuum nisi propter peccatum". De peccatorum meritis et remissione 1,4,1 PL 44,112; voir: Contra sec. Juliani responsionem imp. opus 6,12 PL 45,152.

37. De lib.arb. 3,20,55 PL 32,1297; M. Huftier, Le tragique de la condition chrétienne chez saint Augustin, Paris - Roma 1964, p. 224-227.

38. De civ. Dei 21,3 PL 41,710-712.

39. L. Boros, Mysterium mortis, Freiburg im Br. 1962, p. 9 .
} 
permettent-ils une telle interprétation du phénomène de la mort? Certains pensent que oui ${ }^{40}$. Dans notre conviction, la théorie de "'option finale" ne trouve pas de bases réelles dans sa philosophie et sa théologie.

La théorie mentionnée reconnaît le sens ontologique de la mort. La mort de chaque homme est interprétée comme l'étape de sa plus haute évolution spirituelle. Elle serait, malgré le dépérissement de la vie biologique, un complément dans le pirocessus de l'auto-perfectionnement personnel de l'homme. Alors la mort possède un profil positif, et il faut l'attendre avec joie. Or St. Augustin estime autrement la fonction de la mort. Dans le De Trinitate, en référant les opinions de Cicéron dans son écrit perdu. Hortensius, il écrit: "La mort nous sera douce, à nous qui aurons accompli notre tâche d'homme, et nous nous éteindrons sans regrets: ce sera comme le repos de la vie" ". Cicéron, inspiré par la philosophie des stoiciens, aperçoit dans le phénomène de la mort des aspects positifs. II encourage à l'attendre dans la paix de l'esprit. L'Evêque d'Hippone, malgré toute sa considération pour le penseur romain, polémise avec sa conception de la mort. II écrit notamment: "Je m'étonne qu'un si grand esprit, s'adressant à des hommes adonnés à la philosophie qui rend heureux par la contemplation de la vérité, leur promette que, une fois accomplie leur tâche d'homme, la mort leur sera douce, même si ce qui fait l'objet de nos pensées et de nos affections est mortel et caduc: comme si mourait alors et disparaissait quelque chose que nous n'aimions pas, bien plus, que nous haissions de tout coeur au point de la voir disparaître avec joie" ${ }^{42}$. Dans cette interprétation, la mort n'est pas quelque chose de positif, et c'est ainsi qu'il est difficile de l'attendre avec tranquillité et avec joie. Elle ne donne rien à l'homme, elle est anéantissement de la vie, putréfaction du corps, et relatif non-être.

Dans un pareil esprit, le problème de la mort est discuté dans les autres écrits d'Augustin. II affirme que le plus souvent, l'homme craint la mort. La crainte de la mort n'est nullement le résultat

40. Voir: BA,t.XXXV, p. 515-517. C'est une note anonyme, un commentaire au $13 \mathrm{e}$ livre du De civitate Dei, suggérant la convergence de la théorie de l" option finale" avec les écrits d'Augustin. Et c'est justement cela que nous contestons.

41. Trin. 14,19,26 PL 42,1056; BA,t.XVI; p. 417.

42. Ibidem. 
de préjugés, mais résulte de la nature de l'homme, désirant une existence ultérieure ${ }^{43}$. Dans le De civitate Dei, nous trouvons l'observation, que la mort est le mal de l'homme, car elle est un processus contraire à la vie ${ }^{41}$. Un peu plus loin, la thèse apparaît que la mort n'est bonne pour personne ${ }^{45}$. La manière d'argumentation pour une telle attitude mérite l'attention. Or, la mort - én tant que séparation de l'âme du corus - est un anéantissement de la nature psychophysique de l'homme, souvent elle apporte avec elle les souffrances physiques et psychiques, enfin elle est une interruption brutale de la suite de l'existence humaine. Une telle caractéristique de la mort est expressément négative, c'est pourquoi elle exclut l'acceptation de la théorie de l' "option finale". Cette théorie traite la mort en tant qu'étape de l'évolution la plus haute de la persónnalité humaine. L'Evêque d'Hippone écrit autrement sur le thème de la mort et sur son rôle dans la vie de l'homme. II distingue notamment le processus de "mouirir" du moment final de "la mort". "46. Le premier, l'agonie, est toujours un mal d'existence pour l'homme, cependant la mort comprise comme définitif terme de la vie, est bonne ou mauvaise, en dépendance de la valeur morale et religieuse de toute la vie. La mort des gens bons est définitivement un certain bien, la mort des mauvais est toujours quelque chose de mal: Toute la vie est essentielle dans la relation entre l'homme et Dieu, aussi ne faut-il pas traiter la mort seule comme le moment de la plus haute évolution de la personne humaine. La mort, conçue en elle-même, est toujours un mal ontique. Le sens et le "bien" de la mort s'actualise uniquement en sa liaison avec la vie:

Les écrits de St. Augustin autorisent la conclusión, que la mort de l'homme n'a pas de sens ontique. Ce n'est pás équivalent avec la contestation d'un quelconque sens de la mort; cair ce serait une contestation du sens de l'existence humaine. La mort, bien qu'elle soit un mal ontologique, peut recevoir un sens éthique-eschatologique: Cette approbation du sens de la mort ne se fait pas automati-

43. "Mortem quippe horret, non opinio, sed natura". Sermo 172,1,1 PL 38,936.

44. De civ. Dei 13,4 PL 41,379.

45. Ibidem 13,6 PL 41, 381.

4.6. "Proinde si non dedignaris dicere, quod te cerno nescire, mors in morientibus omnibus mala est; in mortuis autem quibusdam mala, quibusdam bona. Hoc secuti sunt, qui de bono mortis laudabiles disputationes etiam litteris mandaverunt". Contra sec. Juliani responsionem imp. opus 6,27 PL 45,1575 . 
quement, mais dépend de la nature de l'homme, le plus souvent de tout le contexte de sa vie. La bonté de Dieu fait que la mort; présentant un mal -en tant que peine pour le péché- peut enfin se transformer en un bien de l'homme ${ }^{47}$. Caractéristique est l'assertion de St: Augustin. "Mais quelle que soit chez les mourants la douleur qui fait perdre tout sentiment, si elle est pieusement et saintement supportée, elle augḿnente le mérite de la patience, elle ne perd pas le nom de peine" ${ }^{48}$. La mort est donc une peine -un mal, mais reçue en esprit chrétien, elle peut être une occasion à l'approfondissement spirituel. La mort du corps, y inclus l'élément de l'agonie dramatique, est une épreuve de la foi et de la fidélité de l'homme envers Dieu ${ }^{43}$. C'est alors qu'apparaît la patience et: le courage de l'homme, ou bien l'entière faiblesse de son esprit.

Le sens de la mort moral et eschatologique se lie à la reconnaissance de l'inmortalité de l'homme et à la croyance à la résurrection. St. Augustin, en se rapportant aux textes bibliques, affirme que nous verrons Dieu alors "quand nous aurons payé la dette de la mort et reçu la promesse de la résurrection" 50. Si la mort était la fin absolue et définitive de l'homme, alors la vie humaine serait privée de tout espoir motivé et lui donnant un sens. Si cependant l'immatériel "moi" humain ne meurt pas, alors la mort biologique n'annule pas le sens de la personne humaine. "L'âme n'est donc pas une harmonie toute physique. La mort ne peut frapper les êtres immuables. Donc l'âme vit à jamais, soit qu'elle soit ellemême raison, soit que la raison lui soit inséparablement unie" 5i. L'affirmation de l'immortalité est la condition de retrouver le sens éthique-eschatologique dans ce qui est un non-sens ontologique, c'est-à-dire dans la mort.

La théorie de l'“option finale" interprête la mort en tant que le plus adéquat, ou même unique moment de se déclarer pour Dieu. St. Augustin caractérise la nature de la mort d'une manière différente, en différenciant et en opposant la dernière étape de la viẹ

47. Contra duas epist. Pelagianorum 4,3,6 PL 44,613-614.

48. De civ. Dei 13,6 PL 41,381 ; BA,t.XXXV, p. 265.

49. Epist. 157,3 $\mathrm{PL} 33,683 ;$ De peccatorum meritis et remissione 2,32 , 52 - 36,58 PL 44, 182-186.

50. Trin. 2,17,28 PL 42,864.

51. De immort. an. 2,2 PL 32,1022; BA,t,V. Trad. P. Labriolle, Paris 1948, p. 175. Voir: G. Verbeke, Spiritualité et immortalité de l'âme chez saint Augustin, dans: Augustinus Magister, t.I, Paris 1954, p. 329334. 
d'hommes mauvais et d'hommes bons. Le mal de l'existence qu'est la mort se métamorphose en bien moral seulement chez les gens qui savent payer avec le bien pour le mal, qui répondent à la haine par l'amour, qui sont fidè'es jusqu'à la fin à la vérité ${ }^{52}$. La "bonne" mort n'a lieu que lorsqu'elle est précédée d'une bonne vie. Augustin fait une observation amère, que beaucoup de gens ressentent la peur de la mort, mais en même temps ils ne craignent pas de mener une vie mauvaise. Le chrétien doit avant tout se soucier de la bonne vie, et alors chaque genre de mort sera un passage vers un bonheur éternel ${ }^{53}$. Bien que la mort présente toujours un mal d'existence pour la personne humaine, cependant reçue dans l'esprit de l'espérance chrétienne d'une vie éternelle, elle acquiert un sens eschatologique.

\section{L'attitude du chrétien devant la mort}

La mort n'est pas un phénomène accidentel, concernant les individus, mais une perspective perpétuelle de tous. L'homme, en tant qu'ontique union de l'âme et du corps, se trouve toujours devant une réelle possibilité de mort ${ }^{54}$. Cela éveille sa peur, car la nature répugne à l'anéantissement. Lépicuréisme et le stoïcisme, connus d'Augustin, contestaient l'individuelle immortalité de l'homme. Simultanément, ils recommandaient l'attitude d'impassibilité intérieure envers la mort, en suggérant de surmonter la crainte et de se débarrasser de tout désir ${ }^{55}$. Epicure et Epictète affirmaient que ce n'est pas la mort même qui est le plus gran mal, -bien qu'elle soit anéantissement- mais la crainte de la mort. A I'homme, et surtout au philosophe, convient une attitude d'équilibre d'esprit envers la mort, l'acceptation intérieure et "libre" de cette perspective, voire lattente de la mort.

St. Augustin polémise avec une telle "philosophie de la mort".

52. Quaest. in Pentateuchum 7,49 PL 34,811; Contra sec. Juliani respon. imp. opus 6,27 PL $45,1575$.

53. "Mori male times, male vivere non times. Corrige male vivere, time male mori. Sed noli timere: non potest male mori, qui bene vixerit. ...Id age, ut bonam vitam habeas; e quaecumque occasio fuerit ut exeas de hoc corpore, exis ad requiem, exis ad beatitudinem, quae not habet timorem nec finem". De disciplina christiana 12,13 PL 40,676-677.

54. Voir: De civ. Dei 13,9-11 PL 41, 382-385.

55. Epicure, Letter to Menoecus, dans: W.J. Oates, The Stoic and Epicurean Philosophers, . New York 1940, p. 30-31; Epictète, Entretiens, II, 1, ed. J. Souilhé, Paris 1948-1949. 
D'abord, étant sur le terrain du réalisme psychologique, il reconnaît le naturel de la peur humaine. Dans les Confessions, il décrit son prope état d'âme, lorsqu'il était fatigué par la vie, mais simultanément, il craignait la mort ${ }^{56}$. La mort est toujours terrible pour l'homme, qui fuit l'anéantissement de son existence. Nous trouvons une polémique essentielle avec l'attitude épicurienne-stoïcienne dans le livre XIII de l'oeuvre De Trinitate. L'auteur y démontre l'impossibilité de vaincre la peur de la mort dans le cadre de la philosophie du naturalisme et de l'immanentisme; un tel essai est d'ailleurs engagé dans des inconséquences. Plein d'ironie est l'énoncé suivant d'Augustin: "Voilà tout le bonheur-dérisoire s'il n'était pitoyable- de mortels orgueilleux, qui se glorifient de vivre comme ils veulent, parce que c'est volontairement qu'ils supportent avec patience des maux qu'ils voudraient éviter. C'est, dit-on, le sage conseil que donnait Térence: 'Puisque ne peut se réaliser ce que tu veux, il faut vouloir ce que tu peux'. Parole pleine de sens, personne ne le nie; mais conseil donné à un malheureux, pour l'empêcher d'être plus malheureux". ${ }^{57}$. La crainte de la morit est trop naturelle et forte, pour que l'homme soit capable de la désirer sciemment et "ibrement". L'attitude d'une calme distance envers la mort, chère aux stoïciens, ne résoud pas le problème du sens de la vie humaine. La seule domination de l'inquiétude psychique ne donne pas le bonheur à l'homme, elle ne peưt que prévenir un plus grand malheur.

Les méditations suivantes de St. Augustin se réfèrent au fait que la perspective de la mort -en tant que fin de la vie et des valeurs y attenantes - fait l'homme malheureux. Le regard naturaliste sur le phénomène de la mort exclut la définitive résolution du problème, aussi ne donne-t-il pas de bonheur authentique. "Tous ies hommes veulent donc être heureux: s'ils le veulent vraiment, ils veulent aussi, par le fait même, être immortels; autrement, ils ne pourraient être heureux" ${ }^{58}$. Les stoïciens postulaient la conservation du calme intérieur devant la perspective de la mort, mais estce là une proposition réelle? En théorie, chaque homme peut prendre une attitude triple envers le phénomène de la mort: la craindre,

56. "Sed in me nescio quis affectus nimis huic contrarius ortus erat, et taedium vivendi erat in me gravissimum, et moriendi metus". Confes. 4,6,11 PL 32, 697 .

57. Trin. 13,7,10 PL 42,1021; BA,t.XVI, p. 293.

58. Trin. 13,8,11 PL 42,1022; BA,t.XVI; p. 295. 
la. désirer, être impassible. Augustin considère tour à tour les alternations particulières. Si la mort vient contre la volonié de l'homme, alors cela exclut le bonheur. "Si nul n'est heureux qu'il n'ait ce qu'it veut, combien moins encore celui qui se voit enlever contre son gré, non pas les honneurs; les richesses ou toute autre chose, mais la vie heureuse elle-même qu'il perd" " ${ }^{5}$. II faut aussi exclure la deuxième alternative, d'après laquelle l'homme "désire" la mort dii malgré cela, il conserve le bonheur. L'auteur de De Trinitate affirme judicieusement qu'une telle vie qu'on désire perdre, ne peut être définie comme authentiquement heureuse. "Reste la troisième hypothèse: l'homme heureux serait dans l'indifférence, c'est-à-dire perdrait la vie heureuse, quand par la mort il perd toute vie, sans y répugner comme sans le souhaiter, le coeur paisible, prêt à vivre, comme à mourir. Mais cette vie n'est pas non plus vraiment heureuse; qui ne mérite pas l'amour de celui qu'elle rend heureux". ${ }^{60}$. Les analyses de l'Evêque d'Hippone indiquent avec justesse l'impuissance et l'absurdité de la philosophie épicurienne et stoïcienne. L'argumentation verbale et sophiste ne donne pas de base à vaincre la peur de la mort. Si on traite la mort comme entière destrucIion de l'homme, alors il faut en dernier lieu reconnaître le non-sens de la vie humaine. Et la philosophie des non-sens n'est pas la matière indiquée pour former la félicitologie.

Augustin prend une autre attitude envers la mort; une attitude d'espérance, appuyée sur la conviction de l'immortalité du "moi" psychique-intérieur de l'homme. Il aperçoit et il comprend l'existence de la peur de la mort qui approche, mais il la surmonte à l'aide de motifs philosophiques et théologiques. La motivation philosophique de l'attitude d'espérance est liée principalement avec la thèse de l'immortalité de l'âme humaine. Les chrétiens surmontent aussi la peur de la mort, car ils croient à la résurrection future. La foi est la cause que la mort - malgré son horreur - peut être attendue comme le début de la vie étérnelle en unité avec Dieu ${ }^{61}$. La foi vive des premiers martyrs étalt source de courage en vue de la mort et des souffrances liées à elle ${ }^{62}$. La foi en la résurrection s'appuie sur la vérité de la mort et de la résurrection de Jésus-Christ. Le Fils

59. Ibidem.

60. Ibidem PL $42,1022-1023$; BA,t.XVI, p. 297 :

61. Enar. in ps. 102,14 PL 37,1328.

62. De consolatione mortuorum, sermo 2,4-5 PL 40,1164-1166; De peccatorum meritis et remissione 2,31,51-52 PL 44,182-183. 
de Dieu,: "revêtu d'une chair mortelle, ne mourant que par elle," ne ressuscitant que par elle, par elle seule il s'est mis à l'unisson avec nous pour la mort et la résurrection, en se faisant par elle sacrement pour l'homme intérieur et exemple pour l'homme extérieur" ${ }^{63}$. La résurrection du Christ possède une double fonction relativement aux hommes: $1 .^{\circ}$ elle est annonce et "modèle" de notre résurrection, $2 .^{\circ}$ elle est signe de la puissance de Dieu et source de grâce.

La mort est un mal ontologique également pour le chrétien, mais conçue dans le contexte de la mort du Fils de Dieu, elle prend d'autres dimensions. La mort de l'homme est "anéantie" et surmontée par l'offrande de la mort du Christ ${ }^{64}$. Garant de notre résurrection, qui est destruction de la mort, se porte le Christ souffrant et ressuscité. Son agonie douloureuse est encouragement à l'acceptation intérieure de la mort et des souffrances y liées. St. Augustin comprend la crainte de la mort et il s'en accuse lui-même. Mais simultanément il indique que le chrétien devrait sentir la peur devant une "mauvaise" mort, et non devant la mort comme telle. L'homme, en s'intériorisant dans les mystères de Dieu, peut surmonter la peur de la mort. "Et, pour que l'âme soit moins embarrassée d'adherer tout entière à l'intégrale vérité, on désire comme suprême récompense cette mort qu'on redoutait auparavant (la mort) c'est-à-dire la fuite, l'évasion hors de ce corps-ci" ${ }^{66}$. L'attitude d'Augustin chrétien envers le problème de la mort est entièrement différente de ceIle que recommadaient les stoïciens. II n'ignorait ni ne minimalisait pas le fait de la peur de la mort qui accompagne la vie humaine. En même temps, cependant, il donnait des arguments rationnels, philosophiques et théologiques, permettant de surmonter cette peur. L'affirmation de l'immortalité de l'âme et la foi en la résurrection permettent au chrétien d'accepter la mort.

St. Augustin tire de ses analyses la conclusion suivante: $\mathrm{Si}$ la mort est inévitable, il faut donc tâcher que ce soit une "bonne mort". Toujours possible, même à l'heure de la mort, est la réorientation intérieure de l'homme. Mais le plus souvent, la "bonne mort" suit une bonne vie ${ }^{67}$. Eprouver religieusement le mystère de la mort

63. Trin. $4,3,6$ PL 42,891 ; BA,t.XV., p. 351.

64. Trin. $4,2,4$ PL 42,889 .

65. Ibidem 4,3,6 PL 42,891.

66. De quant. an. 33,76 PL 32,1077; BA,t.V, p. 387. Voir: L. Ballay, Der Hoffnungsbegriff bei Augustinus, München 1963, p. 171-280.

67. De civ. Dei 1,11 PL 41,25-26. 
et de la résurrection du Christ est le meilleure préparation à l'entrée dans le mystère de la mort.

L'Evêque d'Hippone attire encore l'attention des vivants sur leurs devoirs envers les morts. Ce sont des devoirs doubles, concernant aussi bien le corps que l'âme. Il existe un devoir d'enseve. lir avec respect le corps du mort; en faveur de ce devoir parlent les raisons autant éthiques et psychologiques que bibliques ${ }^{68}$. Le second devoir élémentaire du chrétien est la prière à l'intention du décédé. Monique, mourante, la demandait à ses fils.

La conception de la mort chez St. Augustin, bien qu'elle contienne des éléments pas assez travaillés mérite l'attention de divers points de vue. D'abord, c'est une heureuse union de la description phénoménologique de la mort avec la profondeur de l'analyse philosophique et théologique de sa nature, son origine et sa fonction. Le trait positif de cette conception est l'intégration du réalisme psychologique à l'optimisme doctrinal modéré. Le penseur chrétien antique a observé la parfois tragique dimension de vivre la mort chez les humains, mais simultanément il a indiqué les prémisses doctrinales autorisant les chrétiens à prendre l'attitude de l'espoir. Caractéristiques en cette matière sont les paroles d'Augustin écrites après la perte de sa mère: "Mais pour ma mère, ce n'était ni une mort misérable, ni une mort totale" " La foi en l'immortaiité et la réssurrection transforme la peur et la douleur de la mort en espoir de l'attente. La solution du problème de la mort, esquissée par l'auteur du De Trinitate, différe essentiellement des principes de la théorie contemporaine de l' "option finale". Le penseur que nous référons, bien qu'il eût vu la gravité du moment de la mort, ne dépréciait cependant pas la totalité de la vie humaine. Uniquement une bonne vie est en état de transformer le mal ontique de la mort en un bien éthique-religieux. Dans l'interprétation de St. Augustin, la mort, bien qu'elle soit sur le plan ontologique une destruction et une privation, cependant, conçue dans le contexte de la mort et de la résurrection du Christ, elle prend un sens eschatologique.

\section{S. KOWALOZYK}

Katolicki Uniwersytet Lubelski

68. Ibidem 1,13 PL 41, 27-28.

69. Confes. $9,12,29$ PL 32,776 . 